\title{
Health Education and Public Health
}

2021; 4(2): 415 - 420. doi: 10.31488/HEPH.163

\section{Research article}

\section{Time Spent by COPD Patients Lying Down during Sedentary Behavior}

\author{
Yuichiro Azuma, Yoshiaki Minakata, Takahiro Kaki, Seigo Sasaki, Kazumi Kawabe, Hideya Ono \\ Department of Respiratory Medicine, National Hospital Organization Wakayama Hospital, Mihama-cho, Japan
}

Corresponding author:Yoshiaki Minakata, Department of Respiratory Medicine, National Hospital Organization Wakayama Hospital, Mihama-cho, Japan, Tel: +81-738-22-3256, Fax: +81-738-22-3104

Received: April 15, 2021; Accepted: May 24, 2021; Published: May 31, 2021

\begin{abstract}
Physical activity is the most relevant risk factor for mortality in patients with chronic obstructive pulmonary disease (COPD), and sedentary time has recently been reported to be an independent risk factor for mortality. We investigated the relationship between the sedentary time and the duration of lying-down or sitting to clarify the details of sedentary behavior in COPD patients using two types of triaxial accelerometers. Sixteen stable COPD patients were included in the analysis. The duration with 1.0-1.5 metabolic equivalents (METs) during waking time was correlated with the lying-down + sitting time but not with the separate lying or sitting time. The duration with 1.0-1.5 METs accounted for $>60 \%$ of both the lying-down and sitting times, and the duration with 0 METs accounted for approximately $30 \%$ of the lying-down time. The ratio of lying-down time to sitting time tended to be low in younger patients and those with low dyspnea. Reducing sedentary time is often argued primarily as shortening the sitting time. However, as the sedentary time in COPD patients consists of both lying and sitting components, it might be important to consider how to reduce not only sitting time but also lying-down time in order to reduce the overall sedentary time.
\end{abstract}

Keywords: Chronic obstructive pulmonary disease (COPD), sedentary, physical activity, METs, lying-down time

\section{Introduction}

Reduced levels of physical activity are associated with a poor prognosis in patients with chronic obstructive pulmonary disease (COPD) [1,2]. Physical activity is the most relevant risk factor for mortality in COPD patients [3]. Recently, sedentary behavior has begun attracting attention, as it is a risk factor for COPD mortality, independent of moderate to vigorous intensity of physical activity [4-12].

Sedentary behavior is defined as all waking behaviors with an energy expenditure of $\leq 1.5$ metabolic equivalents (METs) spent in a sitting or lying posture $[13,14]$. To evaluate the physical activity, a triaxial accelerometer has been widely used [15-21] and research using the Active Style Pro HJA-750C ${ }^{\circledR}$ (HJA; Omron Healthcare, Kyoto, Japan) has been conducted to evaluate the intensity of physical activity in Japan [21-23]. Activity $<1.0$ MET in intensity cannot be detected by an accelerometer and is shown as 0 METs, so sedentary time can be detected by an accelerometer as the duration of activity at 1.0-1.5 METs [24]. As sedentary behavior is defined by the intensity of activity but includes a lying-down or sitting posture [14], the ratio of the types of activity performed during sedentary time may vary from disease to disease.

Replacing 30 minutes of sedentary time with mild or moderate to vigorous intensity of activity reduced mortality in men over 45 years of age [25]. Furthermore, COPD patients with a long TV viewing time were shown to have a higher mortality rate than those with less viewing time [26]. Efforts to reduce sedentary time often focus on shortening the sitting time, but sedentary behavior includes both sitting and lying-down time. In healthy subjects, most sedentary behavior might be performed in a sitting posture; however, the details in COPD patients are unclear.

The present study investigated the rates of sitting and lying down during sedentary time in COPD patients by comparing the relationship between the sedentary time obtained by an HJA, which can detect the intensity of physical activity, and the lying-down or sitting times obtained by a DynaPort MoveMonitor ${ }^{\circledR}$ (DMM; McRoberts BV, The Hague, the Neth- 
erlands), which can detect different types of physical activity.

\section{Materials and Methods}

\section{Patients}

COPD patients $\geq 40$ years of age who visited the National Hospital Organization Wakayama Hospital from March 2017 to September 2017 were recruited. COPD was defined as a post-bronchodilator forced expiratory volume in 1 second (FEV1)/ forced vital capacity (FVC) of $<70 \%$. Patients with clinically apparent bronchial asthma, using oxygen therapy, with a history of lung resection, with a history of an exacerbation of COPD within three months, or with an extremely suppressed physical activity due to diseases other than COPD were excluded.

\section{Protocol}

This was a prospective observational study of a single facility that was conducted at the National Hospital Organization Wakayama Hospital. The HJA can detect the intensity of activity performed each minute and records the values in METs; however, it records any value $<1.0$ MET as "0 METs". The DMM records the durations of five types of activity (lying, sitting, standing, shuffling, and locomotion) in seconds.

The HJA and DMM were worn on the waist at the same time for five days from the time the subject woke up until they went to bed, except for when taking a bath. Among the recorded data, days when the wearing time was $\geq 8 \mathrm{~h}$ were defined as valid days, and the data of the patients for whom $\geq 3$ valid days were obtained were selected for the analysis, based on previous reports $[15,21]$. The wearing time was detected according to the wearing time of the DMM, which was determined by a manufacturer-defined calculation program, and the data from times when the DMM device was not worn were excluded. The average values of data from three valid days of wearing time from the beginning were employed as the representative values of each patient. Three studies were conducted using the valid data obtained.

This study was conducted in accordance with the Declaration of Helsinki and was approved by the local ethics committee (IRB Committee of National Hospital Organization Wakayama Hospital; approval number: 27-8; approval date: March 20, 2015). It was registered with the University Hospital Medical Information Network (UMIN 000016363, January 28, 2015). Written informed consent was obtained from all patients.

1) The relationship between the duration with 1.0-1.5 METs and the lying-down and sitting time.

The duration with 1.0-1.5 METs was calculated as the sum of the time recorded as 1.0-1.5 METs every minute by the HJA, and the lying-down time, sitting time, and total time spent lying and sitting (lying down + sitting) were calculated as the sum of each type of activity, which was determined every second by the DMM. The relationships between the duration of activity at 1.0-1.5 METs, as measured by the HJA, and the time spent lying, sitting, and lying down + sitting (as determined by the DMM) were examined.

2) Rates of activity intensities while lying down and sitting.

To detect the intensity of activity in each posture, only the data in which patients continuously remained lying or sitting for 1 minute (between 0 and 59 seconds of each minute on the clock) were selected. Data obtained when patients had adopted several postures within a minute were excluded. For the ratio of the 0 METs, 1.0-1.5 METs, 1.6-1.9 METs, 2.0-2.9 METs, and $\geq 3.0$ METs times regarding the lying-down time were calculated for each subject. Then the average value of the data of all subjects was then evaluated. The same procedure was also conducted for the sitting time.

3) Relationship between the lying-down/sitting ratio and demographic factors.

To evaluate the factors affecting the ratio of each posture, the ratio of the lying-down time to the sitting time (lying-down/sitting ratio) while awake was compared according to demographic factors, including the age, pack-year, body mass index, modified British Medical Research Council (mMRC), inspiratory capacity, FVC \% of predicted (\%pred), and FEV1 \%pred. The subjects were divided into two groups based on the median value of each factor, and the lying-down/sitting ratios were compared between the two groups.

\section{Statistical analyses}

A correlation coefficient was evaluated for the relationships between the duration of activity at 1.0-1.5 METs and time spent lying, sitting, or lying down + sitting. An unpaired t-test was used to analyze the relationships between the lying-down/sitting ratio and demographic factors.

\section{Results}

Eighteen patients were registered, and two were excluded because they wore accelerometers during both waking and sleeping. At least three valid days of data were obtained for all patients. Ultimately, 16 patients were included in the analysis (Figure 1).

The characteristics of the patients were as follows: males, $\mathrm{n}=15$; females, $\mathrm{n}=1$; age, $74.3 \pm 5.6$ years old; FVC \%pred, $84.3 \% \pm 19.6 \%$; and FEV1 \%pred, $54.9 \% \pm 17.8 \%$ (Table 1). The average time spent wearing the DMM was $748 \pm 111$ minutes. The time spent performing activity of each intensity was as follows: 0 METs, $103 \pm 80$ minutes; $1.0-1.5$ METs, $404 \pm 137$ minutes; 1.61.9 METs, $87 \pm 39$ minutes; 2.0-2.9 METs, $120 \pm 72$ minutes; and

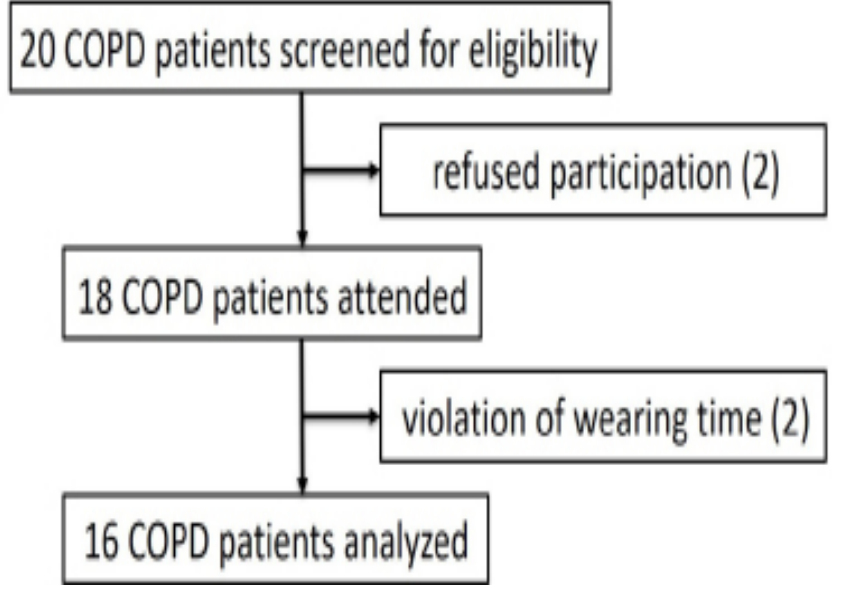

Figure 1. The CONSORT diagram. 
Table 1. Patient caracteristics.

$\begin{array}{lc}\text { sex(male/female) } & 15 / 1 \\ \text { age } & 74.3 \pm 5.6 \\ \text { pack-year } & 85.6 \pm 45.2 \\ \text { BMI } & 19.3 \pm 2.8 \\ \text { mMRC }(0 / 1 / 2 / 3 / 4) & 2 / 6 / 2 / 60 \\ \text { Stage }(\mathrm{I} / \mathrm{II} / \mathrm{III} / \mathrm{IV}) & 1 / 7 / 7 / 1 \\ \text { IC }(\mathrm{L}) & 1.96 \pm 0.36 \\ \text { FVC (L) } & 2.76 \pm 0.57 \\ \text { FVC \%pred (\%) } & 84.3 \pm 19.6 \\ \text { FEV1 (L) } & 1.41 \pm 0.36 \\ \text { FEV1 \%pred (\%) } & 54.9 \pm 17.8 \\ \text { FEV1/FVC (\%) } & 51.5 \pm 9.5\end{array}$

Abbreviations: BMI: body mass index; mMRC: modified British Medical Research Council; IC: Inspiratory capacity; FVC: forced vital capacity; \%pred: \% of predicted; FEV1: Forced expiratory volume in one second.

Table 2. The average time spent performing activity of each intensity or each type of activity.

\begin{tabular}{|lcc|}
\hline & time $(\mathrm{min})$ & $\%$ \\
\hline total & $748 \pm 111$ & 100 \\
\hline Intensity of activity (METs) & & \\
\hline 0 & $103 \pm 80$ & 13.7 \\
\hline $1.0-1.5$ & $404 \pm 137$ & 54.0 \\
\hline $1.6-1.9$ & $87 \pm 39$ & 11.6 \\
\hline $2.0-2.9$ & $120 \pm 72$ & 16.1 \\
\hline$\geq 3.0$ & $34 \pm 25$ & 4.6 \\
\hline type of activity & & \\
\hline lying & $212 \pm 160$ & 28.3 \\
\hline sitting & $370 \pm 123$ & 49.4 \\
\hline standing & $101 \pm 73$ & 13.5 \\
\hline shuffling & $18 \pm 14$ & 2.4 \\
\hline locomotion & $47 \pm 30$ & 6.3 \\
\hline
\end{tabular}

$\geq 3.0$ METs, $34 \pm 25$ minutes. The time spent performing each type of activity was as follows: lying down, $212 \pm 160$ minutes; sitting, 370 \pm 123 minutes; standing, $101 \pm 73$ minutes; shuffling, $18 \pm 14$ minutes; and locomotion, $47 \pm 30$ minutes (Table 2).

Regarding the relationship between the duration with 1.0-1.5 METs and the lying-down and sitting time, no correlation was found between the duration with 1.0-1.5 METs while waking and the time spent lying down $(r=0.382, p=0.145)$ (Figure $2 \mathrm{~A})$ and sitting $(\mathrm{r}=0.296, \mathrm{p}=0.265)$ (Figure 2B). A significant correlation was found between the duration with 1.0-1.5 METs and the time spent lying down + sitting $(r=0.761, p<0.001$; Figure $2 \mathrm{C}$ ). For rates of activity intensities while lying down and sitting, the time spent performing activity of 0 METs and 1.0-1.5 METs accounted for $29.5 \%$ and $62.7 \%$ of the lying-down time, respectively (Figure 3A).

The time spent performing activity of 0 METs, 1.0-1.5 METs, and $\geq 1.6$ METs accounted for $9.2 \%, 63.3 \%$, and $27.5 \%$ of the sitting time, respectively (Figure 3B). Considering relationship between the lying-down/sitting ratio and demographic factors, when the patients were grouped according to the median values of each factor, the lying-down/sitting ratio did not differ markedly for any of the factors (Table 3). However, in the younger group and the group with a better mMRC, the lying-down/sitting ratio tended to be smaller than in others $(\mathrm{p}=$ $0.227, \mathrm{p}=0.216$, respectively), and the lying-down time tended to be shorter.

\section{Discussion}

In COPD patients, a correlation was observed between the duration with 1.0-1.5 METs while awake and the lying down + sitting time but not between the duration with 1.0-1.5 METs and the lying-down or sitting times (Figure 2). In terms of the intensity of activity spent while lying down and sitting, the duration with 1.0-1.5 METs accounted for more than $60 \%$ of both the lying-down and sitting times (Figure 3). The duration with 0 Mets accounted for approximately $30 \%$ of the lying-down time, and the duration with $\geq 1.6$ Mets accounted for approximately $30 \%$ of the sitting time.

A correlation was found between the duration with 1.0-1.5 METs and the lying down + sitting time but not between the duration with 1.0-1.5 METs and the lying-down or sitting times (Figure2). This indicates that both the lying-down and sitting times were equally important factors in the sedentary time of COPD patients. Previous reports on sedentary behavior have recommended reducing TV-viewing time or breaking up long sitting times to reduce the sedentary time [26-28]. There has thus been a focus on how to reduce sitting time. As both lying-down and sitting times can influence the sedentary time in COPD patients, reducing the sedentary time might require considering reducing not only the sitting time but also the lying-down time. As the intensity of activity while lying down was lower than that while sitting - as seen in the results of Study 2-reducing lying-down time might be more important than reducing sitting time in COPD patients.

The lying-down time accounted for $28 \%$ of the total waking time per day, while the sitting time accounted for $49 \%$ of the total time (Table2). Pitta et al. reported that the lying-down and sitting times accounted for $12 \%$ and $52 \%$ of the total waking time in COPD patients, respectively [15]. Furlanetto et al. reported that the lying-down and sitting times accounted for $14 \%$ and $44 \%$ of the total waking time, respectively [4]. In the current study, the percentage of time spent sitting was similar to that in previous reports, while the percentage of time spent lying down was longer than in those reports. The FEV1\%pred in this study was rather high (current study, $54.9 \% \pm 17.8 \%$; previous studies, $43 \% \pm 18 \%$ or $41 \%$ ), and the average age of the patients in the current study was relatively old (current study, 74 years old; previous study, 64 or 66 years old) (Table1). Godfrey et al. reported that healthy subjects of 70-75 years of age had a significantly longer sedentary than those 65-70 years of age [28]. COPD patients also might indulge in longer sedentary times as they become older. Considering the results of our study, wherein the percentage of sitting time was the same as in previous studies while the rate of lying-down time was longer than in previous reports, the sedentary time, especially the lying-down time, might increase as patients age, although the lying-down/sitting ratio 


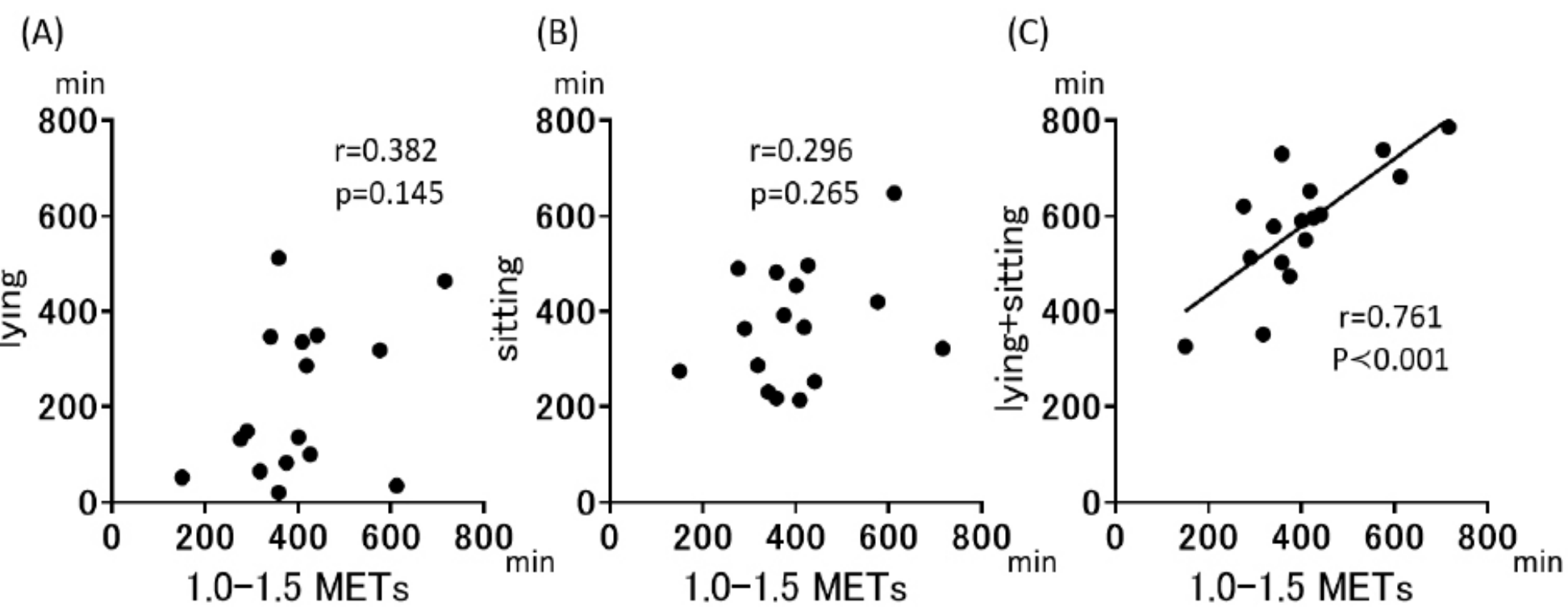

Figure 2. The relationship between the duration with 1.0-1.5 METs, as measured by the HJA, and the time spent lying down (A), sitting (B), and lying down + sitting $(C)$, as measured by the DMM.

(A) lying

(B) sitting

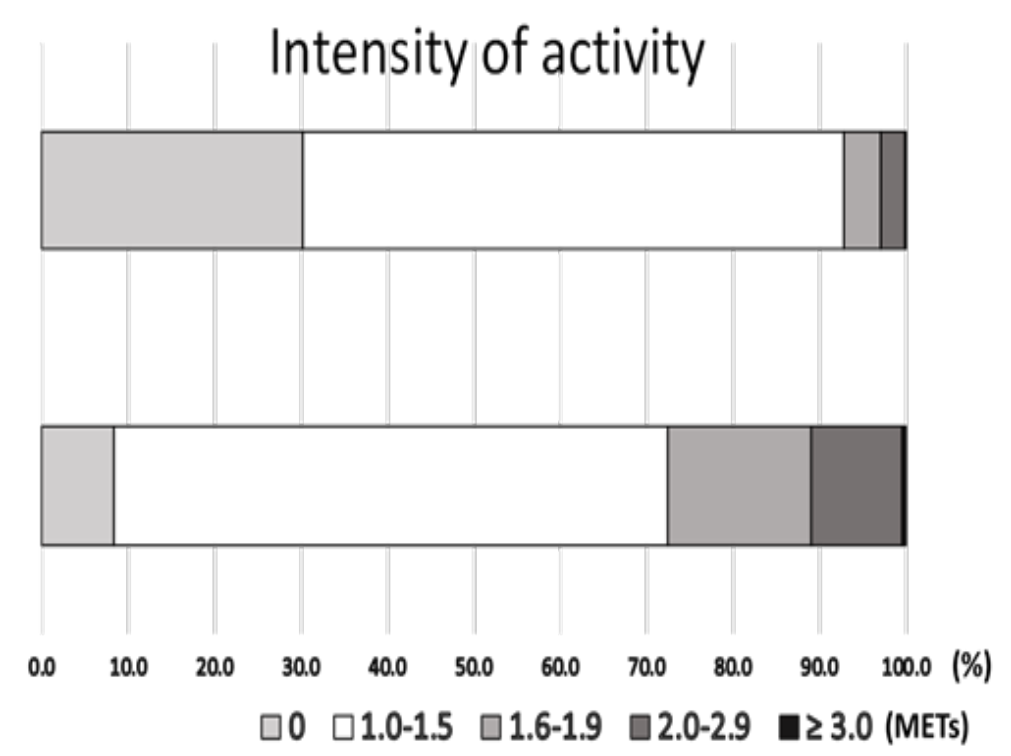

Figure 3. The percentage of time spent performing activities of each intensity while lying down (A) and sitting (B). 
Table3. Therelationshipsbetweenthelying/sittingratioanddemographicfactors.

\begin{tabular}{|c|c|c|c|c|}
\hline & lying(min) & sitting(min) & lying/sitting & $p$ \\
\hline age & & & & 0.227 \\
\hline$<73.5$ & $154 \pm 125$ & $405 \pm 145$ & $0.51 \pm 0.58$ & \\
\hline$\geq 73.5$ & $269 \pm 178$ & $334 \pm 94$ & $0.81 \pm 2.16$ & \\
\hline pack-year & & & & 0.318 \\
\hline$<84$ & $177 \pm 172$ & $408 \pm 135$ & $0.55 \pm 0.61$ & \\
\hline$\geq 84$ & $245 \pm 150$ & $330 \pm 105$ & $0.91 \pm 0.78$ & \\
\hline $\mathrm{BMI}$ & & & & 0.709 \\
\hline$<19$ & $217 \pm 174$ & $380 \pm 154$ & $0.80 \pm 0.72$ & \\
\hline$\geq 19$ & $206 \pm 156$ & $359 \pm 94$ & $0.66 \pm 0.72$ & \\
\hline mMRC & & & & 0.216 \\
\hline$<2$ & $163 \pm 160$ & $400 \pm 127$ & $0.51 \pm 0.57$ & \\
\hline$\geq 2$ & $214 \pm 134$ & $354 \pm 130$ & $0.95 \pm 0.79$ & \\
\hline IC & & & & 0.882 \\
\hline$<2 \mathrm{~L}$ & $190 \pm 178$ & $378 \pm 145$ & $0.52 \pm 1.62$ & \\
\hline$\geq 2 \mathrm{~L}$ & $216 \pm 119$ & $396 \pm 125$ & $0.65 \pm 1.55$ & \\
\hline FVC \% pred & & & & 0.339 \\
\hline$\geq 80 \%$ & $154 \pm 144$ & $365 \pm 143$ & $0.56 \pm 0.61$ & \\
\hline$<80 \%$ & $209 \pm 115$ & $400 \pm 109$ & $0.90 \pm 0.78$ & \\
\hline FEV1 \% pred & & & & 0.504 \\
\hline$\geq 50 \%$ & $181 \pm 135$ & $357 \pm 99$ & $0.61 \pm 0.58$ & \\
\hline$<50 \%$ & $243 \pm 186$ & $382 \pm 150$ & $0.85 \pm 0.83$ & \\
\hline
\end{tabular}

was not significantly associated with age in the current study.

The lying-down time was 212 minutes, the sitting time was 370 minutes, and the lying-down/sitting ratio was 0.57 in the present study (Table2). The lying-down/sitting ratio was not correlated with any demographic factors; however, it tended to be high in the older group and in the high mMRC group. Pitta et al. reported that the lying-down/sitting ratio was 0.10 in healthy subjects and 0.23 in COPD patients [15]. The lying-down/sitting ratio in healthy subjects was much smaller than that in COPD patients. The sedentary time in healthy subjects might be largely composed of the sitting time, while that in COPD patients might include a higher rate of lying-down time. This suggests that reducing the lying-down time may become an important target for reducing sedentary time in COPD patients. Although the lying-down time and sitting time have often been considered together as the sedentary time, it may be important to focus on the lying-down time when discussing sedentary behavior in the future studies of COPD patients.

Regarding the rates of the intensities of activity during lying down and sitting, the time spent engaged in activity of 1.01.5 METs in intensity accounted for more than $60 \%$ of the time during both lying and sitting (Figure3). This indicates that both lying-down and sitting times are equally important elements of sedentary behavior in COPD patients. Activity of $\geq 1.6$ Mets, which is beyond the category of sedentary behavior, was hardly observed during the lying-down time, but approximately $30 \%$ of the sitting time was spent performing activity of this intensity. An intensity of activity in the sitting time was relatively high, while activity of this intensity was rarely performed in lying-down time. Thus, reducing the lying-down time together with reducing the sitting time may be important for reducing the sedentary time in COPD patients.

Some of the lying-down time during waking may be recorded as 0 METs, which cannot be detected as sedentary time when an intensity-type accelerometer is employed. Although sedentary behavior is defined as all behavior of $\leq 1.5$ METs during waking hours, the duration of activity of $<1.0$ MET cannot be detected. In the current study, the time spent performing activity of 0 METs accounted for approximately $30 \%$ of the total lying-down time while awake, which means that activity was not detected by an accelerometer for approximately $30 \%$ of the lying-down time. Thus, when the lying-down time is evaluated, undetected lying-down time (about 30\%) should be considered.

Several limitations associated with the present study warrant mention. First, the number of patients was relatively small. A further study with a larger number of patients is required to evaluate the effect of the lying-down time. Second, the time that the subjects spent wearing the DMM differed among the measured days, as the waking time differs from day to day. We were only able to confirm whether or not the HJA had been worn at the same time as the DMM was worn by asking the patients, and we were unable to exclude data obtained when subjects were napping. Unfortunately, these problems are difficult to solve.

\section{Conclusion}

The sedentary time in COPD patients consists equally of both lying-down and sitting components. Among the sedentary time, lying-down time is relatively long. To reduce the sedentary time, it is important to consider how to reduce not only sitting time but also lying-down time.

\section{Acknowledgements}

Authors thank Mr. Brian Quinn for reading manuscript.

\section{Conflicts of Interest}

The authors declare no conflict of interest.

\section{References}

1. Garcia-Aymerich J, Lange P, Benet M, et al. Regular physical activity reduces hospital admission and mortality in chronic obstructive pulmonary disease: a population based cohort study. Thorax. 2006;61:772-8.

2. Garcia-Rio F, Rojo B, Casitas R, et al. Prognostic value of the objective measurement of daily physical activity in patients with COPD. Chest. 2012;142:338-46.

3. Waschki B, Kirsten A, Holz O, et al. Magnussen. Physical activity is the strongest predictor of all-cause mortality in patients with COPD: a prospective cohort study. Chest. 2011; 140:331-42.

4. Furlanetto KC, Donária L, Schneider LP, et al. Sedentary Behavior Is an Independent Predictor of Mortality in Subjects with COPD. Respir Care. 2017;62:579-87.

5. Cavalheri V, Straker L, Gucciardi DF, et al. Changing physical activity and sedentary behaviour in people with COPD. Respirol.2016;21(3):419-26.

6. Geidl W, Carl J, Cassar S, et al. Physical Activity and Sedentary Behaviour Patterns in 326 Persons with COPD before Starting a Pulmonary Rehabilitation: A Cluster Analysis. J Clin Med. 2019; 29;8(9):1346.

7. Wshah A, Selzler AM, Hill K, et al. Determinants of Sedentary Behaviour in Individuals with COPD: A Qualitative Exploration Guided by the Theoretical Domains Framework.COPD.2020;17(1):6573. 
8. Lewthwaite H, Effing TW, Lenferink A, et al. Improving physical activity, sedentary behaviour and sleep in COPD: perspectives of people with COPD and experts via a Delphi approach. PeerJ. 2018;6:e4604.

9. Bernard P, Hains-Monfette G, Atoui S, et al. Daily Objective Physical Activity and Sedentary Time in Adults with COPD Using Spirometry Data from Canadian Measures Health Survey. Can Respir J.2018:9107435.

10. McKeough ZJ, Large SL, Spencer LM, et al. An observational study of self-reported sedentary behaviour in people with chronic obstructive pulmonary disease and bronchiectasis. Braz J Phys Ther.2020;24(5):399-406.

11. Alyami MM, Jenkins SC, Hill K. Walking-based activity and sedentary behavior in Saudi males with chronic obstructive pulmonary disease. Saudi Med J. 2018;39(5):506-513.

12. Hill K, Gardiner PA, Cavalheri V, et al. Physical activity and sedentary behaviour: applying lessons to chronic obstructive pulmonary disease. Intern Med J. 2015;45(5):474-82.

13. Sedentary Behaviour Research Network. Letter to the editor: standardized use of the terms "sedentary" and "sedentary behaviours". Appl Physiol Nutr Metab. 2012;37:540-2

14. Tremblay MS, Aubert S, Barnes JD, et al. Sedentary Behavior Research Network (SBRN) - Terminology Consensus Project process and outcome. Int J Behav Nutr Phys Act 2017;14:75

15. Pitta F, Troosters T, Spruit MA, et al. Characteristics of physical activities in daily life in chronic obstructive pulmonary disease. Am J Respir Crit Care Med. 2005;171:972-7.

16. Rabinovich RA, Louvaris Z, Raste Y, et al. Validity of physical activity monitors during daily life in patients with COPD. Eur Respir J. 2013;42:1205-15.

17. Gimeno-Santos E, Raste Y, Demeyer H, et al. The PROactive instruments to measure physical activity in patients with chronic obstructive pulmonary disease. Eur Respir J. 2015;46:988-1000.

18. Langer D, Gosselink R, Sena R, et al. Validation of two activity monitors in patients with COPD. Thorax. 2009;64:641-2.

19. Hill K, Dolmage T.E, Woon L, et al. Measurement properties of the SenseWear armband in adults with chronic obstructive pulmo- nary disease. Thorax. 2010;65:486-91.

20. Sugino A, Minakata Y, Kanda M, et al. Validation of a compact motion sensor for the measurement of physical activity in patients with chronic obstructive pulmonary disease. Respiration. 2012;83:300-7.

21. Miyamoto S, Minakata Y, Azuma Y, et al. Verification of a Motion Sensor for Evaluating Physical Activity in COPD Patients. Can Respir J. 2018;8343705.

22. Ichinose M, Minakata Y, Motegi T, et al. Efficacy of tiotropium/ olodaterol on lung volume, exercise capacity, and physical activity. Int J Chron Obstruct Pulmon Dis. 2018;13:1407-19.

23. Minakata Y, Motegi T, Ueki J, et al. Effect of tiotropium/olodaterol on sedentary and active time in patients with COPD: post hoc analysis of the VESUTO((R)) study. Int J Chron Obstruct Pulmon Dis. 2019;14:1789-801.

24. Pate RR, O'Neill JR, Lobelo F. The evolving definition of "sedentary". Exerc Sport Sci Rev. 2008;36:173-8

25. Diaz KM, Duran AT, Colabianchi N, et al. Potential Effects on Mortality of Replacing Sedentary Time With Short Sedentary Bouts or Physical Activity: A National Cohort Study. Am J Epidemiol. 2019;188:537-44.

26. Ukawa S, Tamakoshi A, Yatsuya H, et al. Association Between Average Daily Television Viewing Time and Chronic Obstructive Pulmonary Disease-Related Mortality: Findings From the Japan Collaborative Cohort Study. J Epidemiol 2015;25:431-6.

27. Katzmarzyk PT, Church TS, Craig CL, et al. Sitting time and mortality from all causes, cardiovascular disease, and cancer. Med Sci Sports Exerc 2009;41:998-1005.

28. Stephens SK, Eakin EG, Clark BK, et al. What strategies do deskbased workers choose to reduce sitting time and how well do they work? Findings from a cluster randomised controlled trial. Int J Behav Nutr Phys Act. 2018;15:98.

29. Godfrey A, Lord S, Galna B, et al. The association between retirement and age on physical activity in older adults. Age Ageing. 2014;43:386-93.

To cite this article: Azuma Y, Minakata Y, Kaki T. Time Spent by COPD Patients Lying Down during Sedentary Behavior. Health Education and Public Health. 2021; 4:2.

(C) 2021 Azuma Y, et al. 